\title{
Amaranth oil reduces accumulation of 4- hydroxynonenal-histidine adducts in gastric mucosa and improves heart rate variability in duodenal peptic ulcer patients undergoing Helicobacter pylori eradication
}

Andriy Cherkas, Kamelija Zarkovic, Ana Cipak Gasparovic, Morana Jaganjac, Lidija Milkovic, Orest Abrahamovych, Ostap Yatskevych, Georg Waeg, Olha Yelisyeyeva \& Neven Zarkovic

To cite this article: Andriy Cherkas, Kamelija Zarkovic, Ana Cipak Gasparovic, Morana Jaganjac, Lidija Milkovic, Orest Abrahamovych, Ostap Yatskevych, Georg Waeg, Olha Yelisyeyeva \& Neven Zarkovic (2018) Amaranth oil reduces accumulation of 4-hydroxynonenal-histidine adducts in gastric mucosa and improves heart rate variability in duodenal peptic ulcer patients undergoing Helicobacter pylori eradication, Free Radical Research, 52:2, 135-149, DOI: 10.1080/10715762.2017.1418981

To link to this article: https://doi.org/10.1080/10715762.2017.1418981

Accepted author version posted online: 17 Dec 2017. Published online: 18 Jan 2018.

LII Article views: 192

View Crossmark data $[\pi$
Submit your article to this journal $[\pi$

Q View related articles $\sqsubset$

Citing articles: 6 View citing articles 지 


\title{
Amaranth oil reduces accumulation of 4-hydroxynonenal-histidine adducts in gastric mucosa and improves heart rate variability in duodenal peptic ulcer patients undergoing Helicobacter pylori eradication
}

\author{
Andriy Cherkas $^{\mathrm{a}}$ (D), Kamelija Zarkovic ${ }^{\mathrm{b}}$, Ana Cipak Gasparovic ${ }^{c}$, Morana Jaganjac $^{\mathrm{d}}$ (D), Lidija Milkovic ${ }^{\mathrm{c}}$ (D), \\ Orest Abrahamovych ${ }^{\mathrm{a}}$ (D) Ostap Yatskevych ${ }^{\mathrm{a}}$ (D), Georg Waeg ${ }^{\mathrm{e}}$, Olha Yelisyeyeva ${ }^{\mathrm{f}}$ and Neven Zarkovic ${ }^{\mathrm{C}}$

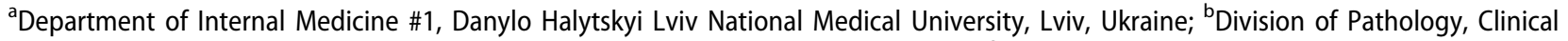 \\ Hospital Centre "Zagreb", University of Zagreb School of Medicine, Zagreb, Croatia; 'Laboratory for Oxidative Stress (LabOS), Institute

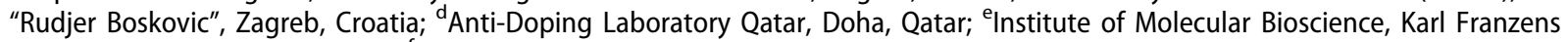 \\ University of Graz, Graz, Austria; 'Department of Histology, Cytology and Embryology, Danylo Halytskyi Lviv National Medical \\ University, Lviv, Ukraine
}

\begin{abstract}
Helicobacter pylori-induced oxidative stress in gastric mucosa (GM) is a milieu for the development of chronic gastritis, duodenal peptic ulcer (DPU), gastric cancer, and a number of extragastric diseases. Because our previous study revealed the accumulation of the protein adducts of lipid peroxidation product 4-hydroxynonenal (HNE) in GM, which persists after eradication of $H$. pylori, the aim of the study was to test whether Amaranth oil supplementation in addition to standard anti-Helicobacter treatment could prevent such accumulation of HNE in GM in $H$. pyloripositive DPU patients. Seventy-five patients were randomly split into two groups: group 1 standard treatment $(n=39)$ and group 2 - standard treatment with additional supplementation of $1 \mathrm{ml}$ of concentrated oil from amaranth seeds (Amaranthus cruenthus L., $n=36$ ). Clinical analysis, including endoscopy with biopsies from antrum and corpus of the stomach were performed before and after the treatment, as was heart rate variability (HRV) recorded, as parameter of systemic, extragastric pathophysiological alterations in DPU patients. Improvement of clinical, endoscopic and histologic manifestations, and successful ulcer healing were observed in both the groups. Moreover, supplementation of amaranth oil in addition to standard anti-H. pylori treatment significantly reduced accumulation of HNE-histidine adducts in GM and increased HRV in DPU patients $(p<.05)$. Therefore, standard treatments of DPU require additional therapeutic approaches, in accordance with integrative medicine principles, aiming to reduce persistence of oxidative stress, as was successfully done in our study by the use of amaranth oil.
\end{abstract}

Abbreviations: AO: Amaranth oil; DPU: duodenal peptic ulcer; GM: gastric mucosa; HF: high frequency; HNE: 4-hydroxy-2-nonenal; H. pylori: Helicobacter pylori; HRV: heart rate variability; LF: low frequency; OS: oxidative stress; OT: orthostatic test; pNN50: percentage of differences between adjacent normal RR intervals exceeding 50 milliseconds; RMSSD: square root of the mean squared differences of successive RR intervals; ROS: reactive oxygen species; SDNN: standard deviation of normal RR intervals; TP: total power; VLF: very low frequency

\section{ARTICLE HISTORY}

Received 22 September 2017 Revised 24 November 2017

Accepted 13 December 2017

\section{KEYWORDS}

Peptic ulcer; oxidative stress; Helicobacter pylori; amaranth oil; 4-hydroxynonenal; heart rate variability

\section{Introduction}

Duodenal peptic ulcer (DPU) is one of the most common diseases of gut and its complications include lifethreatening conditions such as perforation, penetration, and bleeding. The link between Helicobacter pylori and DPU, as well as with chronic gastritis, gastric cancer, and MALT-lymphoma is well established [1]. A growing evidence shows that $H$. pylori may be associated with the so-called "extra-gastric manifestations" such as insulin resistance, metabolic syndrome, nonalcoholic fatty liver disease, atherosclerosis, autoimmune, and neurodegenerative diseases, etc. [2,3]. Recent large cohort study demonstrated positive association of H. pylori-positivity with body mass index, a major risk factor of metabolic diseases, regardless socioeconomic status and other cofounders [4], clearly indicating substantial systemic metabolic effects of $H$. pylori infection. Fortunately, eradication of $H$. pylori was proven to be effective treatment of chronic gastritis and peptic ulcer, and potentially could be effective in the prevention of gastric cancer and other diseases associated with this microorganism $[5,6]$.

CONTACT Andriy Cherkas cherkasandriy@yahoo.com @ Department of Internal Medicine \#1, Danylo Halytskyi Lviv National Medical University, Lviv, Ukraine 
Helicobacter pylori is known to be able to induce local damage to gastric epithelial cells and cause inflammation in gastric mucosa (GM). This is associated with migration of neutrophils and development of gastritis. It is assumed that chronic gastritis with or without the ulcer may significantly contribute to systemic effects of $H$. pylori. Excessive generation of reactive oxygen species (ROS) induced by microorganism's virulence factor, predominantly CagA in GM and oxidative burst caused by activated neutrophils leads to the development of local oxidative stress (OS) [3]. It is also possible that local OS and low-grade inflammation caused by persistence of $H$. pylori in human stomach are major contributors to its extragastric manifestations $[7,8]$. Released into circulation tissue degradation products as well as multiple regulatory molecules may exert significant systemic effects. However, intensity of inflammation should be strong enough to overcome detoxifying function of liver, which serves as a natural "filter" for all kinds of substances coming from the gut $[8,9]$.

Oxidative stress is often associated with increased accumulation of lipid peroxidation products, among which 4-hydroxy-2-nonenal (HNE) is one of the most prominent [10]. HNE is considered to be a bioactive marker of pathological processes and a "second messenger" of ROS [11]. Since HNE is highly reactive, it is difficult to detect free HNE in the organism while it often covalently binds to proteins thus producing conjugates mainly with cysteine, histidine, and lysine residues [12], while the HNE-protein adducts can be detected by immunohistochemistry [12] and ELISA [13]. These assays still remain a state of the art of the HNE detection in biological samples and are greatly contributing to modern understanding of the role of HNE as biomarker of OS [14].

As we previously demonstrated, $H$. pylori causes accumulation of the histidine adducts of HNE in GM of DPU patients. At the same time some degree of HNE accumulation in $G M$, but significantly less than in patients, was also observed in some of apparently healthy $H$. pylori-negative and positive volunteers that also participated in the study [15]. However, eradication of $H$. pylori did not cause significant reduction of HNE accumulation, 4 weeks after eradication revealing persistence of OS in GM after treatment [16]. This is also in consistence with the evidence from randomised controlled studies that eradication of $H$. pylori does not reduce short-term risk of gastric cancer, while reduction of its risk is gained only after 8-10 years after eradication, while some show even the lack of significant effects of $H$. pylori eradication [5].

It remains unknown whether only the presence of H. pylori is sufficient for causing systemic effects, or active inflammatory process is required for that [17]. In this regard, the study focused on the determination of inflammatory parameters, HNE-water soluble end-metabolite and 8-iso-prostaglandin $\mathrm{F} 2-\alpha$ as measures of OS in apparently healthy $H$. pylori-negative and positive sedentary subjects did not reveal significant differences [18]. However, the changes were observed when heart rate variability (HRV) in these subjects was analysed [8]. Decreased HRV, despite the lack of clear evidence of direct link, was long associated with OS-related diseases [19,20]. Indirect evidence that the interventions aimed to reduce OS could lead to improvement of HRV also support this observation [16,21]. This is particularly important, since modulation of autonomic control substantially influences microcirculation, which is critical for normal gastric mucosa function. Besides, psychological stress is additional major risk factor and can serve as a trigger for DPU development in subjects with the presence of other risk factors, for example $H$. pylori [22], hence, the HRV is a "gold standard" for noninvasive evaluation of degree of stress and autonomic control of internal organs [23].

Since recently possible links between autonomic regulation and redox signalling are attracting attention of a growing number of researchers. The directions of these studies include anti-inflammatory effects of cholinergic (parasympathetic) signalling [24,25], interactions of adrenergic pathways with $\mathrm{H}_{2} \mathrm{O}_{2}$-mediated insulin signalling [26] and thermogenesis in adipose tissue [27]. It was reported recently that selective Nrf2 deletion in the rostral ventrolateral medulla evokes hypertension and "sympathoexcitation" in mice pointing out to close relationships of autonomic regulation and redox balance [28].

There are many interventions aimed to reduce OS manifestations in experimental and clinical setups. Principal approaches include integrative medicine approaches including pharmacological treatments, supplementation diet by nutraceuticals or even nutritional deprivation (caloric restriction or intermittent fasting), physiotherapy, and exercise [29]. It turned out that, initially considered as "antioxidant", some interventions were shown to be in fact prooxidant; however, their positive effects were achieved by the stimulation of redox signalling and activation of "protective" redox sensitive transcription factors such as Nrf2 or FOXO [30], which stimulate the expression of antioxidant enzymes (thus having hormetic effects). Among other popular, natural products used, the amaranth oil (AO) has a unique composition and is long believed to have beneficial health effects. A number of studies performed on animal models as well as some human 
studies demonstrated a great potential of $\mathrm{AO}$ in modulation of OS in different conditions including high-performance sports [21], diabetes, cancer, etc. In addition, the potential of $A O$ to restore reduced HRV was also demonstrated. The information available in the literature on chemical composition of Amaranth grains including lipid fraction was recently reviewed [31]. Potentially active substances, including carotenoids, tocopherols and tocotrienols, squalene, and polyunsaturated fatty acids [32] present in significant concentrations in $\mathrm{AO}$ are capable to modulate redox signalling, may be responsible for these effects; however, the effects of other substances present at lower concentrations cannot be excluded as well [31].

Since OS is a major problem in DPU and there is no evidence of reduction of HNE-histidine adducts in GM after successful $H$. pylori eradication, it may be assumed that supplementation of AO may have beneficial effect on gastric mucosa when administered orally. This is consistent with the integrative medicine approaches to treat DPU by the holistic protocols including nutraceutical or plant-based treatments [33] as well. In this intervention study, we aimed to test whether oral supplementation of $\mathrm{AO}$ in addition to standard treatment (triple therapy) will have an impact on the accumulation of HNE-histidine adducts and histological parameters in GM, clinical manifestations and HRV in patients with DPU 4 weeks after standard treatment.

\section{Patients, materials and methods}

\section{Study design and recruitment of patients}

Study design is presented in Figure 1. All patients underwent routine clinical examinations and laboratory investigations according to existing protocols and standards of treatment, after signing the informed consent. Examination plan included upper gastrointestinal endoscopy with biopsy from antrum and body of stomach for histological examination (routine histology and $H$. pylori determination), fast urease test, and HNEimmunohistochemistry. H. pylori was determined in 75 patients (age $32.0 \pm 0.8,46$ (61.3\%) male and 29 (34.7\%) females). Endoscopy was performed before and after the treatment as described below (day 0 and day 35).

Standard treatment was prescribed according to the recommendations of second Maastricht consensus (2000) and included lansoprazole $30 \mathrm{mg}$, amoxicillin $1000 \mathrm{mg}$, and clarithromycin $500 \mathrm{mg}$ twice per day for 7 days. In posteradication period patients were asked to take one capsule of lansoprazole $30 \mathrm{mg}$ for 4 weeks before sleep considering that normal time of ulcer healing is 3-4 weeks. There were two main time points: day 0 (a day before the beginning of treatment, usually a

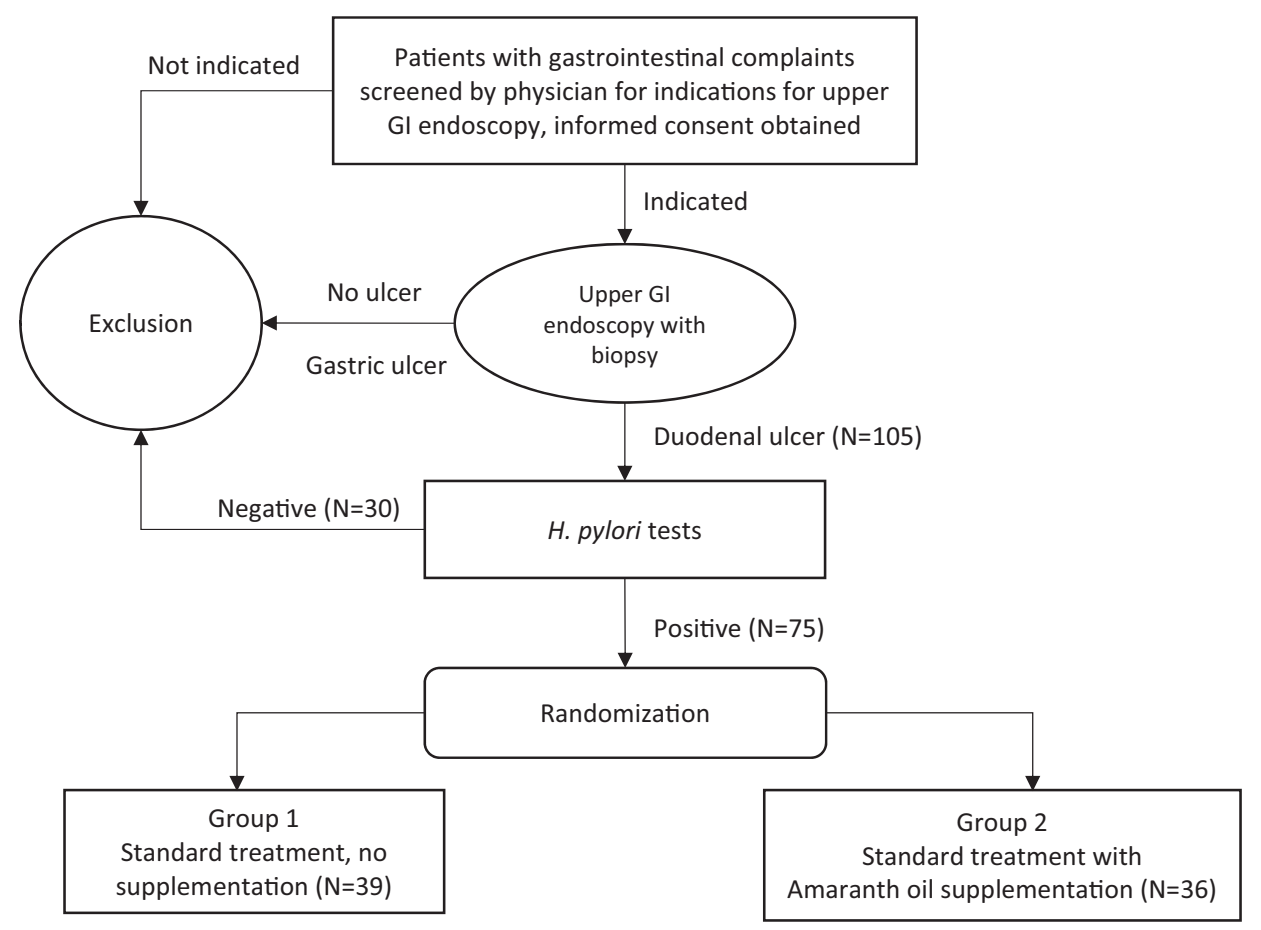

Figure 1. Scheme of recruitment of patients in the study. It includes screening of subjects of respective age with presence of gastrointestinal complaints typical for duodenal ulcer, absence of diseases, symptoms or signs of exclusion criteria. Subjects with H. pylori-positive duodenal ulcers were randomised into study groups. 
day of endoscopy) and day 35 (4 weeks after completion of eradication treatment and a day of second endoscopy). HRV was determined on these days.

All H. pylori-positive subjects were split into two groups: group 1 was recommended standard treatment as described above and group 2 received in addition to standard treatment $A O$ supplementation from day 8 to day $35 \quad(1 \mathrm{ml}$ of concentrated oil derived from Amaranthus cruenthus seeds by the method of $\mathrm{CO}_{2}$ extraction, AO was provided by NVF "Danika", Kharkiv, Ukraine). Oil was taken orally once daily at least $30 \mathrm{~min}$ before breakfast. Patients were provided with sufficient amount of oil and were instructed how to take it. All patients recruited in the study assigned to group 2 reported no compliance failures (failing to take at least six doses of $A O$ in 6 days per respective week in appropriate time).

Control group consisted of 40 gender and agematched healthy subjects without any clinically manifested health disorders or complaints related to gastrointestinal or cardiovascular diseases. These subjects underwent upper gastrointestinal endoscopy with acquisition of gastric mucosa samples once at the beginning of the study. Patients with $\mathrm{H}$. pylori-negative DPU were excluded from the main part of the study after confirmation of absence of the microorganism. Neither $\mathrm{H}$. pylori-negative patients nor control group subjects were prescribed anti-Helicobacter treatment or AO. All recruited patients and apparently healthy subjects participated in the study voluntarily, were aware of required procedures and potential risks. Informed consent was obtained in all screened subjects. The protocol of the study was approved by institutional ethics committee of Danylo Halytskyi Lviv National Medical University before the beginning of any of the procedures of the study.

\section{Histology, immunohistochemistry, and determination of $\mathrm{H}$. pylori}

For histological examination two biopsy specimens were obtained endoscopically from antrum and two from corpus of the stomach in all DPU patients as it is known that $H$. pylori lives predominantly in these gastric parts. As this microorganism is rarely observed in the duodenum, no samples from this region were obtained while the risk of complications (perforation, haemorrhage) is much higher. The biopsy specimens were immediately fixed in buffered $10 \%$ formalin, then dehydrated in ethanol and embedded in paraffin, cut into $5-\mu \mathrm{m}$ thick sections and examined with haematoxylineosin and modified Giemsa staining. Histologically, H. pylori infection was considered as negative if $H$. pylori was absent in all biopsies obtained from one patient and positive if it was in at least one sample. Additionally, one antral biopsy specimen was taken for rapid urease test. Inflammation, presence of $H$. pylori, glandular atrophy, and intestinal metaplasia were classified qualitatively as negative (0) or positive (1); and semiquantitatively into four grades according to updated Sydney System as follows: 0, none; 1, mild; 2, moderate, and 3, severe. Representative paraffin blocks obtained only from patients with successful eradication of $H$. pylori before and after treatment were further used for immunohistochemical staining: one slice of each specimen was cut into $5-\mu \mathrm{m}$ thin sections, mounted on a slide coated with (3-aminopropyl) triethoxysilane, deparaffinated in xylene, and rehydrated through a series of ethanol.

Immunohistochemistry for HNE-modified proteins was carried out as described before $[15,16]$ using monoclonal antibodies obtained from culture medium of clone "HNE 1g4" produced by the fusion of Sp2-Ag8 myeloma cells with B-cells of a BALB-c mouse immunised with HNE-modified keyhole limpet haemocyanin [34]. The antibody is specific for the HNE-histidine epitope in HNE-protein (peptide) conjugates and gives only $5 \%$ cross-reactivity with HNE-lysine and $4 \%$ with HNE-cysteine. Immunohistochemistry was done in a three-step procedure as described before using LSAB kit (DAKO, Denmark) where the first step was incubation with anti-HNE-histidine monoclonal antibodies (dilution 1:10) for 2 hrs in humid chambers at room temperature. The second step was incubation with biotinylated secondary goat antimouse and antirabbit immunoglobulins (AB2) for $30 \mathrm{~min}$. The third step was incubation with streptavidin-peroxidase for $30 \mathrm{~min}$. Finally, the reaction was visualised with DAB (3.3-diaminobenzidine tetrahydrochloride in organic solvent; DAKO, Denmark) after 10 min of incubation. Negative control was done on one histological slice of the same tissue, without application of the HNE-histidine specific monoclonal antibodies. The intensity and distribution of the HNE-immunostaining in the gastric mucosa from antrum and corpus of the stomach were evaluated semiquantitatively. The absence of immunopositivity in the cytoplasm and nuclei of glandular epithelial cells was marked with (0), while with (1) we marked weak immunopositivity in less than $25 \%$ of the cells, with (2) medium immunopositivity in $25-50 \%$ of cells and, finally, with (3) strong immunopositivity in more than $50 \%$ of cells. In superficial and foveolar epithelium as well as in lamina propria of the gastric mucosa we distinguished only positive (1) and negative (0) immunostaining. All immunohistochemical analyses were done by a pathologist experienced in the HNE immunohistochemistry without prior knowledge of the study group design. 


\section{Heart rate variability}

Briefly, during quiet wakefulness in the morning hours, not less than 24 hours after the last significant physical exercise, the short-time records of ECG were performed. A computer electrocardiograph "VNS-Micro" (Neurosoft@, Ivanovo, Russia) was used for ECG records. After 20 min of rest, studied subjects were asked to stay supine quietly for $5.0 \mathrm{~min}$ to ensure quasi-stationary condition HRV recording; afterwards they were asked to stand up rapidly and remained in the standing position for $6 \mathrm{~min}$ - active orthostatic test (OT). RR intervals were determined with a sampling frequency of $2.0 \mathrm{kHz}$ and were analysed with "Poly-Spectrum" (Neurosoft ${ }^{\circledR}$, Ivanovo, Russia) software designed according to HRV standards 7. The time-domain parameters - standard deviation of normal RR intervals (SDNN), the square root of the mean squared differences of successive RR intervals (RMSSD), and percentage of differences between adjacent normal RR intervals exceeding 50 milliseconds ( $p N N 50$ ) were determined. The power spectral analysis was performed sequentially with a fast Fourier transformation. The following frequency-domain variables were studied: total power (TP, $0.01-0.40 \mathrm{~Hz}$ ), high frequency power (high frequency (HF), $0.15-0.40 \mathrm{~Hz}$ ), low frequency power (low frequency $(L F), 0.04-0.15 \mathrm{~Hz})$, and very low frequency power (very low frequency (VLF), 0.01-0.04 Hz). For more details about the procedure and method of short period HRV recording please refer the reference [35].

\section{Statistical analysis}

All data were processed using the statistical package IBM SPSS Statistics 24 (Armonk, NY). Normal distribution of the obtained data was confirmed with Shapiro-Wilk's $W$-test. Normally distributed data was presented as means \pm standard error mean $(\mathrm{M} \pm \mathrm{m})$ and abnormally distributed parameters (most of the parameters of HRV) as median (quartile range). The descriptive data defined a positive/negative is presented as number of positive and \% of total. Nonparametric Mann-Whitney U-test was used to compare HRV parameters and semi-quantitative data, chi-square test was used for qualitative data presented as positive/negative. In figures, data are presented as mean and standard error mean (error bar). If not indicated otherwise, $p<.05$ was considered as significant.

\section{Results}

\section{Clinical symptoms and endoscopy}

The most common symptom of DPU is epigastric pain, which was observed in 35 (89.7\%) and 34 (94.4\%)
Table 1. The frequencies of principal clinical symptoms in $H$. pylori-positive DPU patients before and after treatment, the absolute $(n)$ and respective relative values (\%) are presented.

\begin{tabular}{|c|c|c|c|c|}
\hline \multirow[b]{2}{*}{ Symptom } & \multicolumn{2}{|c|}{ Before treatment } & \multicolumn{2}{|c|}{ After treatment } \\
\hline & $\begin{array}{l}\text { Group } 1 \\
(n=39)\end{array}$ & $\begin{array}{l}\text { Group } 2 \\
(n=36)\end{array}$ & $\begin{array}{l}\text { Group } 1 \\
(n=39)\end{array}$ & $\begin{array}{l}\text { Group } 2 \\
(n=36) \\
\end{array}$ \\
\hline \multicolumn{5}{|l|}{ Complaints } \\
\hline Pain (in epigastrium) & $35(89.7 \%)$ & $34(94.4 \%)$ & $4(10.3 \%)^{c}$ & $2(5.6 \%)^{c}$ \\
\hline Pyrosis & $12(30.8 \%)$ & $13(36.1 \%)$ & $3(7.7 \%)^{b}$ & $1(2.8 \%)^{\mathrm{C}}$ \\
\hline Eructations with acid & $15(38.5 \%)$ & $14(38.9 \%)$ & $1(2.6 \%)^{c}$ & $0(0.0)^{c}$ \\
\hline Nausea & $17(43.6 \%)$ & $15(41.7 \%)$ & $2(5.1 \%)^{c}$ & $1(2.8 \%)^{\mathrm{c}}$ \\
\hline Vomiting & 7 (17.9\%) & $4(11.1 \%)$ & $0(0.0 \%)^{b}$ & $0(0.0 \%)^{a}$ \\
\hline Flatulence & $10(25.6 \%)$ & $4 \%)$ & $3(7.7 \%)^{a}$ & $.6 \%)$ \\
\hline ions & $15(38.5 \%)$ & $12(33.3 \%)$ & $4(10.3 \%)^{b}$ & $1(2.8 \%)^{c}$ \\
\hline Diarrhoea & $3(7.7 \%)$ & $8 \%)$ & $1(2.6 \%)$ & $0(0.0 \%)$ \\
\hline \multicolumn{5}{|l|}{ Physical examination } \\
\hline Coated tongue & $15(38.5 \%)$ & $13(36.1 \%)$ & $6(15.4 \%)^{a}$ & $3(8.3 \%)^{b}$ \\
\hline Halitosis & $13(33.3 \%)$ & $.0 \%)$ & $4(10$. & $2(5.6 \%)^{\mathrm{a}}$ \\
\hline Painful epigastric palpation & $37(94.9 \%)$ & $36(100.0 \%)$ & $1(2.6 \%)^{c}$ & $0(0.0 \%)^{c}$ \\
\hline \multicolumn{5}{|c|}{$\begin{array}{l}\text { aifference between before and after treatment in respective group is } \\
\text { significant by } \gamma^{2} \text { test } p<.05 \text {. }\end{array}$} \\
\hline \multicolumn{5}{|c|}{$\begin{array}{l}{ }^{b} \text { Difference between before and after treatment in respective group is } \\
\text { significant by } \chi^{2} \text { test } p<.01 \text {. }\end{array}$} \\
\hline \multicolumn{5}{|c|}{$\begin{array}{l}\text { 'Difference between before and after treatment in respective group is } \\
\text { significant by } \chi^{2} \text { test } p<.001 \text {. }\end{array}$} \\
\hline \multicolumn{5}{|c|}{$\begin{array}{l}\text { There was no significant difference detected between group } 1 \text { and } 2 \\
\text { neither before nor after the treatment }(p>.05) \text {. }\end{array}$} \\
\hline
\end{tabular}

patients in groups 1 and 2, respectively. Less frequent symptoms include pyrosis, eructation with acid, vomiting, flatulence, constipation, and diarrhoea (Table 1).

Treatment provided radical improvement of patients' condition and in most patients all the symptoms disappeared (Table 1) or were observed only occasionally. We did not observe significant differences between group 1 and group 2 after treatment ( $p>.05$, Table 1$)$. Similarly, the frequency of symptoms detected during physical examination (coated tongue, halitosis, and painful epigastric palpation) significantly decreased after treatment, while indicated differences between the groups were not significant (Table 1). Along with ulcers (present in 100\% of patients included in groups 1 and 2) an endoscopic evidence of gastritis and duodenitis was obtained for almost all patients (Table 2). Other findings included gastroesophageal reflux, endoscopic evidence of reflux esophagitis, duodeno-gastral reflux and the presence of scar in the duodenum, as a result of healing of ulcers in prior periods (Table 2). After treatment, significant improvement of majority of endoscopic symptoms was observed and complete or almost complete ulcer healing was reported. In case of duodenal reflux and scar deformation the difference before and after treatment was not significant, as were no significant differences between groups 1 and 2 .

\section{H. pylori and histology of GM samples (comparison of DPU patients and control group)}

Helicobacter pylori contamination in control group (apparently healthy age- and gender-matched subjects 
Table 2. Endoscopic changes detected in DPU patients before and after treatment, the absolute $(n)$ and respective relative values (\%) are presented.

\begin{tabular}{lcccrr}
\hline & \multicolumn{2}{c}{ Before treatment } & & \multicolumn{2}{c}{ After treatment } \\
\cline { 2 - 3 } \cline { 5 - 6 } Finding & Group 1 $(n=39)$ & Group 2 $(n=36)$ & & Group 1 $(n=39)$ & Group 2 $(n=36)$ \\
\hline Gastritis & $37(94.9 \%)$ & $35(97.2 \%)$ & & $10(25.6 \%)^{\mathrm{c}}$ & $8(22.2 \%)^{\mathrm{c}}$ \\
Duodenitis & $39(100.0 \%)$ & $36(100.0 \%)$ & & $9(23.1 \%)^{\mathrm{c}}$ & $7(19.4 \%)^{\mathrm{c}}$ \\
Gastroesophageal reflux & $13(33.3 \%)$ & $13(36.1 \%)$ & & $5(12.8 \%)^{\mathrm{a}}$ & $3(8.3 \%)^{\mathrm{b}}$ \\
Reflux oesophagitis & $7(17.9 \%)$ & $10(27.8 \%)$ & & $3(7.7 \%)$ & $2(5.6 \%)^{\mathrm{a}}$ \\
Duodenogastral reflux & $17(43.6 \%)$ & $15(41.7 \%)$ & & $8(20.5 \%)^{\mathrm{a}}$ & $8(22.2 \%)$ \\
Scar deformation of duodenum & $14(35.9 \%)$ & $12(33.3 \%)$ & & $14(35.9 \%)$ & $12(33.3 \%)$ \\
\hline
\end{tabular}

${ }^{a}$ Difference between before and after treatment in respective group is significant by $\chi^{2}$ test $p<.05$.

${ }^{\mathrm{b}}$ Difference between before and after treatment in respective group is significant by $\chi^{2}$ test $p<.01$.

'Difference between before and after treatment in respective group is significant by $\chi^{2}$ test $p<.001$.

There was no significant difference detected between group 1 and 2 neither before nor after the treatment.

Table 3. H. pylori contamination and the results of histological examination of gastric mucosa in apparently healthy subjects (control group) and DPU patients (including $H$. pylori-negative), the absolute $(n)$, and respective relative values (\%) are presented.

\begin{tabular}{lccr}
\hline Parameter & $\begin{array}{c}\text { Control group } \\
(n=40)\end{array}$ & $\begin{array}{c}\text { DPU patients } \\
(n=105)\end{array}$ & $\begin{array}{c}\text { Difference by } \\
\chi^{2} \text { test, } p \text { value }\end{array}$ \\
\hline Antrum & & & \\
H. pylori & $16(40.0 \%)$ & $75(71.4 \%)^{\mathrm{c}}$ & $<.001$ \\
Neutrophils & $4(10.0 \%)$ & $24(22.9)$ & .061 \\
Monocytes & $15(37.5 \%)$ & $85(81.0 \%)^{\mathrm{c}}$ & $<.001$ \\
Atrophy & $0(0.0 \%)$ & $10(9.5 \%)^{\mathrm{a}}$ & .043 \\
Metaplasia & $0(0.0 \%)$ & $4(3.8 \%)$ & .211 \\
Dysplasia & $0(0.0 \%)$ & $6(5.7 \%)$ & .123 \\
Lymphoid follicles & $3(7.5 \%)$ & $37(35.2 \%)^{\mathrm{c}}$ & $<.001$ \\
Corpus of stomach & & & \\
H. pylori & $14(35.0 \%)$ & $67(63.8 \%)^{\mathrm{b}}$ & .004 \\
Neutrophils & $4(10.0 \%)$ & $15(14.3 \%)$ & .494 \\
Monocytes & $15(37.5 \%)$ & $77(73.3 \%)^{\mathrm{c}}$ & $<.001$ \\
Atrophy & $0(0.0 \%)$ & $17(16.2 \%)^{\mathrm{b}}$ & .007 \\
Metaplasia & $0(0.0 \%)$ & $2(1.9 \%)$ & .379 \\
Dysplasia & $0(0.0 \%)$ & $0(0.0 \%)$ & 1.000 \\
Lymphoid follicles & $3(7.5 \%)$ & $25(23.8 \%)^{\mathrm{a}}$ & .026 \\
\hline Dyfference between &
\end{tabular}

${ }^{\mathrm{a} D i f f e r e n c e ~ b e t w e e n ~ t h e ~ g r o u p s ~ s i g n i f i c a n t ~ w i t h ~} p<.05$.

${ }^{\mathrm{b}}$ Difference between the groups significant with $p<.01$.

'Difference between the groups significant with $p<.001$.

without gastrointestinal complaints) was detected in 16 subjects (40\%), while, naturally, in DPU patients contamination was significantly higher $(71.4 \% ; p<.001)$. $\mathrm{H}$. pylori was detected in 75 patients, as shown in Table 3. Monocytic infiltration was clearly associated with $H$. pylori positivity and chronic inflammation in GM. It was the case in almost all the subjects, both in apparently healthy controls and in DPU patients (Table 3).

However, neutrophils were observed only in some samples of $H$. pylori-positive subjects and were associated with more severe inflammation either acute or exacerbation of the chronic inflammation (Table 3). The results of histological examination in antrum and corpus of stomach were generally consistent with slightly more pronounced inflammatory changes observed in antrum (pylorus), H. pylori's "favourite" region (Table 3). Lymphoid follicles were also more frequently find in DPU patients, especially in antrum $(p<.001$, Table 3$)$. We did not observe significant difference in degree of
Table 4. The distribution of $H$. pylori-positive apparently healthy subjects and H. pylori-positive DPU patients by degree of microbial contamination of gastric mucosa, the absolute $(n)$ and respective relative values (\%) are presented.

\begin{tabular}{lcr}
\hline $\begin{array}{l}\text { Degree of } H . \text { pylori } \\
\text { contamination }\end{array}$ & $\begin{array}{c}\text { Control group of } \\
\text { apparently healthy } \\
\text { subjects }(n=16)\end{array}$ & $\begin{array}{c}\text { DPU patients } \\
(n=75)^{\mathrm{a}}\end{array}$ \\
\hline II & $8(50.0 \%)$ & $44(58.7 \%)$ \\
III & $5(31.3 \%)$ & $19(25.3 \%)$ \\
a The difference between the groups is insignificant & $3(18.8 \%)$ & $15(20.0 \%)$ \\
\hline
\end{tabular}

microbial contamination of $H$. pylori between apparently healthy subjects and DPU patients, $p>.05$. Moreover, control group showed slightly higher aggregate rates of second and third degrees compared to DPU patients, and more than $50.0 \%$ of DPU patients had low degree of $H$. pylori contamination (Table 4). This suggests that the presence of $H$. pylori in stomach mucosa itself is probably more important factor than the degree of its contamination, while the outcome of this pathogen-host interaction depend lot on the host's intrinsic resistance, metabolic efficiency, and flexibility.

\section{H. pylori and histology of GM samples of DPU patients before and after treatment}

Treatment aimed to eradicate $H$. pylori lead to marked improvement of histological pattern of GM specimens. Efficiency of eradication according to histological evaluation was found to reach 92.5 and $94.4 \%$ in groups 1 and 2, respectively, with $p<.001$ in both cases (Table 5). A strong reduction of inflammatory signs (accumulation of neutrophils, monocytes, and lymphoid follicles) was observed to be associated with elimination of infection, however, in some cases, especially in group 1 accumulation of leucocytes, mainly monocytes, remained (difference between the groups after treatment $p<.01$ in antrum and $p<.05$ in corpus, Table 5). The rates of atrophy, metaplasia, and dysplasia did not 
Table 5. Helicobacter pylori contamination and the results of histological examination of gastric mucosa in DPU patients before and after treatment, the absolute $(n)$ and respective relative values (\%) are presented.

\begin{tabular}{|c|c|c|c|c|}
\hline & \multicolumn{2}{|c|}{ Before treatment } & \multicolumn{2}{|c|}{ After treatment } \\
\hline & Group 1 & Group 2 & Group 1 & Group 2 \\
\hline \multicolumn{5}{|l|}{ Antrum } \\
\hline H. pylori & 39 (100.0\%) & $36(100.0 \%)$ & $3(7.75)^{c}$ & $2(5.6 \%)^{c}$ \\
\hline Neutrophils & $12(30.8 \%)$ & $13(36.1 \%)$ & $1(2.6 \%)^{c}$ & $0(0.0 \%)^{c}$ \\
\hline Monocytes & 38 (97.4\%) & $36(100.0 \%)$ & $12(30.8 \%)^{c}$ & $2(5.6 \%)^{c, e}$ \\
\hline Atro & $4(10.3 \%)$ & $4(11.1 \%)$ & $4(10.3 \%)$ & $1(2.8 \%)$ \\
\hline Met & $2(5.1 \%)$ & $1(2.8 \%)$ & $2(5.1 \%)$ & $0(0.0 \%)$ \\
\hline & $2(5.1 \%)$ & $2(5.6 \%)$ & $1(2.6 \%)$ & $0(0.0 \%)$ \\
\hline Lymphoid follicles & 15 (38.5\%) & 11 (30.6\%) & $3(7.7 \%)^{b}$ & $0(0.0 \%)^{c}$ \\
\hline \multicolumn{5}{|c|}{ Corpus of the stomach } \\
\hline H. pylori & $36(92.3$ & $32(88$ & $2(5.1 \%)^{c}$ & $1(2.8 \%)^{c}$ \\
\hline Neutrophils & $12(30.8 \%)$ & $10(27.8 \%)$ & $1(2.6 \%)^{c}$ & $0(0.0 \%)^{c}$ \\
\hline & 37 (94.9\%) & 31 (86.1\%) & $8(20.5 \%)^{c}$ & $1(2.8 \%)^{c, d}$ \\
\hline & $5(1$ & 70 & $4(10.3 \%)$ & $4(11.1 \%)$ \\
\hline $\mathrm{Me}$ & $2(5.1 \%)$ & $1(2.8 \%)$ & $2(5.1 \%)$ & $0(0.0 \%)$ \\
\hline Dysplasia & $0(0.0)$ & $0(0.0 \%)$ & $0(0.0 \%)$ & $0(0.0 \%)$ \\
\hline Lymphoid follicles & 13 (33.3\%) & $9(25.0 \%)$ & $3(7.7 \%)^{c}$ & $0(0.0 \%)^{b}$ \\
\hline
\end{tabular}

${ }^{\mathrm{b}}$ The difference between before and after treatment significant by $\chi^{2}$ test. $p<.01$ in respective group.

'The difference between before and after treatment significant by $\chi^{2}$ test, $p<.001$ in respective group.

${ }^{\mathrm{d}}$ The difference between the groups significant by $\chi^{2}$ test, $p<.05$.

${ }^{\mathrm{e}}$ The difference between the groups significant by $\chi^{2}$ test, $p<.01$. differ significantly between the groups and remained at relatively low level (Table 5).

\section{Accumulation of 4-hydroxynonenal-histidine adducts in gastric mucosa}

Individual variations of the appearance of the HNEhistidine adducts were observed in both patients and in control group, however, there was a clear evidence that accumulation of HNE in $H$. pylori-positive DPU patients was higher than in $H$. pylori-negative and $H$. pylori-positive apparently healthy subjects in antrum (Figure 2(A,B)) and corpus (Figure 3(A,B)). In the control group, mild and occasional accumulation of $\mathrm{HNE}$ was observed in superficial and foveolar epithelium of antrum of the stomach, while in corpus these cells were found to be free of HNE. In lamina propria, accumulation of HNE was observed in $35.0 \%$ cases in antrum and $40.0 \%$ in corpus of stomach of subjects from control group (Figures 2(C),3(C)). The HNE immunopositivity in glandular epithelium was detected in almost all studied healthy subjects and patients
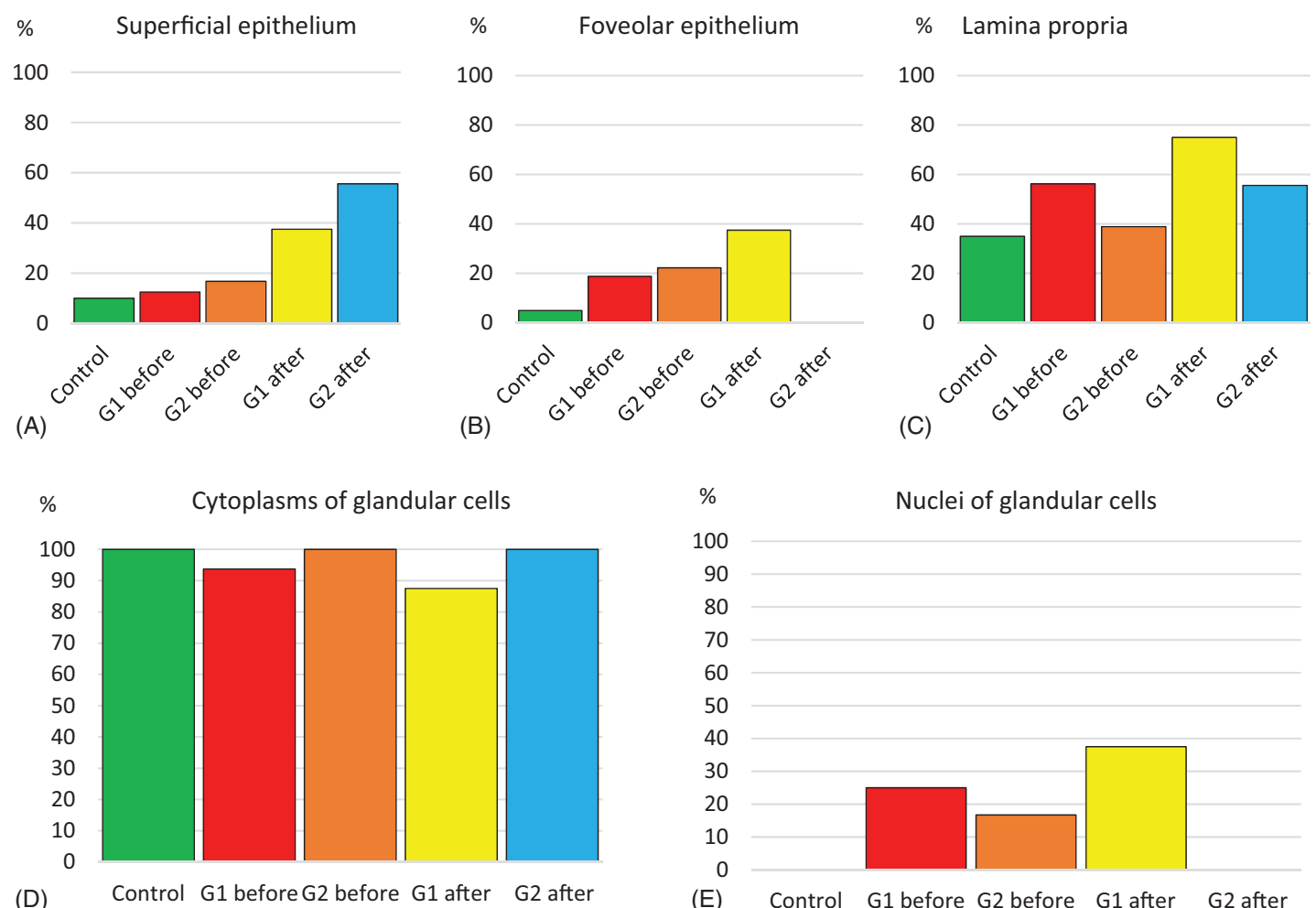

(D)

Control G1 before G2 before G1 after G2 after

(E) Control G1 before G2 before G1 after G2 after

Figure 2. Accumulation of 4-hydroxynonenal-histidine adducts in gastric mucosa of antrum of studied subjects (qualitative evaluation, expressed as \% of cases with respective HNE-immunopositivity of samples). Statistical evaluation by Chi-square test (nonsignificant not indicated): (A) Control vs. G1 after $p<.01$, Control vs. G2 after $p<.0001, \mathrm{G} 1$ before vs. G1 after $p<.01$, G1 before vs. G2 after $p<.001, \mathrm{G} 2$ before vs. G1 after $p<.05$, G2 before vs. G2 after $p<.01, \mathrm{G} 1$ after vs. G2 after $p, 01$; (B) Control vs. G2 before $p<.05$, Control vs. G1 after $p<.001$, G1 before vs. G1 after $p<.01$; G1 before vs. G2 after $p<.01$, G2 before vs. G1 after $p<.05, \mathrm{G} 2$ before vs. G2 after $p<.01, \mathrm{G} 1$ after vs. G2 after $p<.001$; (C) Control vs. G1 after $p<.001$, G2 before vs. G1 after $p<.01$; (D) all nonsignificant; (E) Control vs. G1 before $p<.01$; Control vs. G2 before $p<.05$, Control vs. G1 after $p<.001$; G1 before vs. G2 after $p<.01, \mathrm{G} 2$ before vs. $\mathrm{G} 1$ after $p<.05$, G2 before vs. $\mathrm{G} 2$ after $p<.05, \mathrm{G} 1$ after vs. $\mathrm{G} 2$ after $p<.001$. 


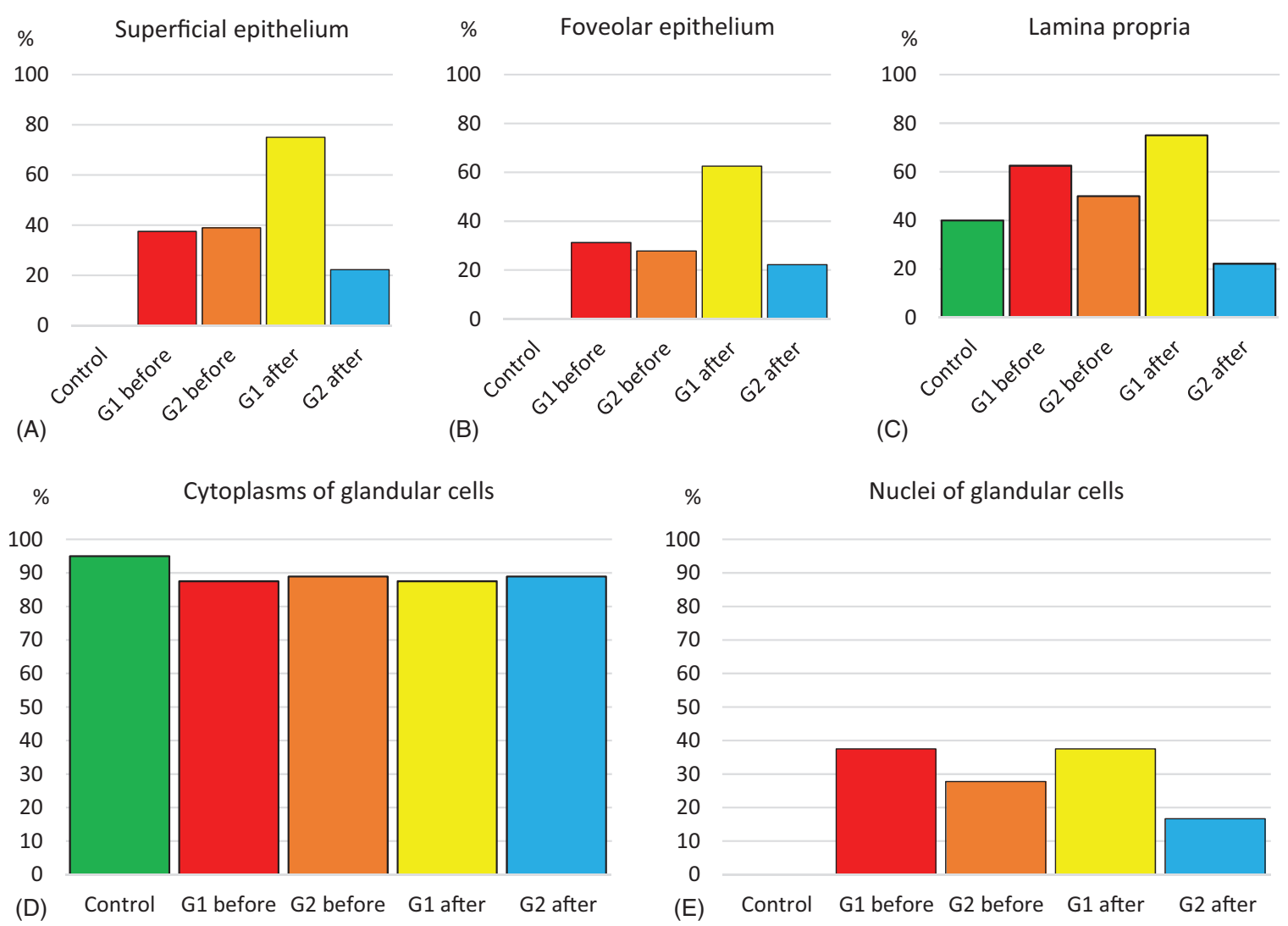

Figure 3. Accumulation of 4-hydroxynonenal-histidine adducts in gastric mucosa of corpus of stomach of studied subjects (qualitative evaluation, expressed as \% of cases with respective HNE-immunopositivity of samples). Statistical evaluation by Chi-square test (nonsignificant not indicated): (A) Control vs. G1 before $p<.001$, Control vs. G2 before $p<.001$, Control vs. G1 after $p<.001$, Control vs. G2 after $p<.001, \mathrm{G} 1$ before vs. G1 after $p<.01, \mathrm{G} 2$ before vs. G1 after $p<.01, \mathrm{G} 1$ after vs. G2 after $p, .001$; (B) Control vs. G1 before $p<.001$, Control vs. G2 before $p<.001$, Control vs. G1 after $p<.001$, Control vs. G2 after $p<.01$, G1 before vs. G1 after $p<.01, \mathrm{G} 2$ before vs. G1 after $p<.01, \mathrm{G} 1$ after vs. G2 after $p, 001$; (C) Control vs. G1 after $p<.01$, G1 before vs. G2 after $p<.001, \mathrm{G} 2$ before vs. G1 after $p<.05, \mathrm{G} 2$ before vs. G2 after $p<.001$, G1 after vs. G2 after $p<.001$; (D) all nonsignificant; (E) Control vs. G1 before $p<.001$; Control vs. G2 before $p<0.001$, Control vs. G1 after $p<.001$.

before and after treatment (statistical difference not significant, $p>.1$ ), however, the pattern of this accumulation was different.

The HNE immunopositivity of cytoplasm of glandular cells was observed almost in all subjects, while the nuclei of granulocytes in samples obtained from control subjects did not show accumulation of HNE-histidine adducts. At the same time, in considerable number of patients besides cytoplasmic accumulation, the HNE immunopositivity in substantial number of samples the accumulation of HNE-histidine adducts in nuclei was also observed (Figures 2(D), 3(D)). Standard treatment did not cause significant reduction of HNE immunopositivity of nuclei of glandular cells in antrum and in corpus of the stomach (Figures 2 and 3). In contrast to the standard treatment, AO supplementation provided significant reduction of nuclear HNE accumulation in DPU patients in both antrum and corpus of the stomach (Figures 2(E) and 3(E)).
In order to study in more details the pattern of HNE accumulation in glandular cells, we performed semiquantitative evaluation of HNE immunopositivity as described in methods section (Figure 4). Despite the evidence that HNE immunopositivity in glandular cytoplasm was common finding and is observed almost in all subjects the intensity (degree) of HNE-protein accumulation was different. The patients before treatment had higher rate of grade 3 (the most severe) HNE immunopositivity in cytoplasm of glandular cells compared to controls (for statistical evaluations see figure captions). Standard treatment did not change this pattern significantly in antrum (Figure 4(A)), while in corpus the rate of severe accumulation of HNE-histidine adducts further increased (Figure 4(C)). Similar findings were observed in nuclei of glandular cells - there was a tendency of increase in grade 3 HNE immunopositivity of nuclei in both antrum and corpus of the stomach (Figures 4(B,D)). 


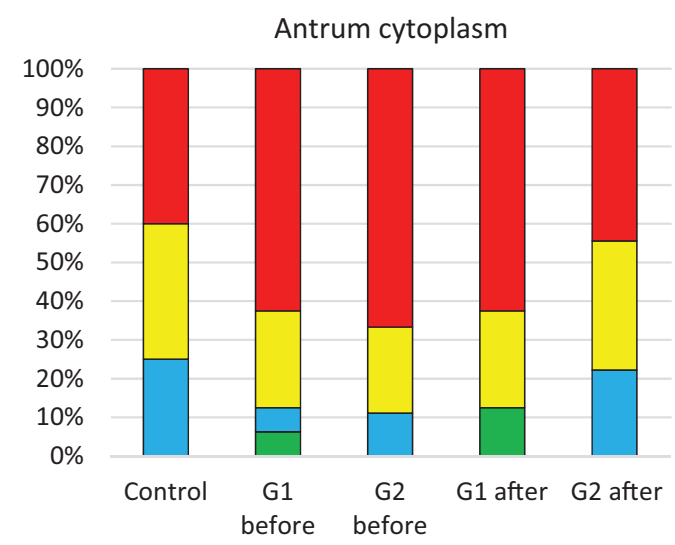

(A)

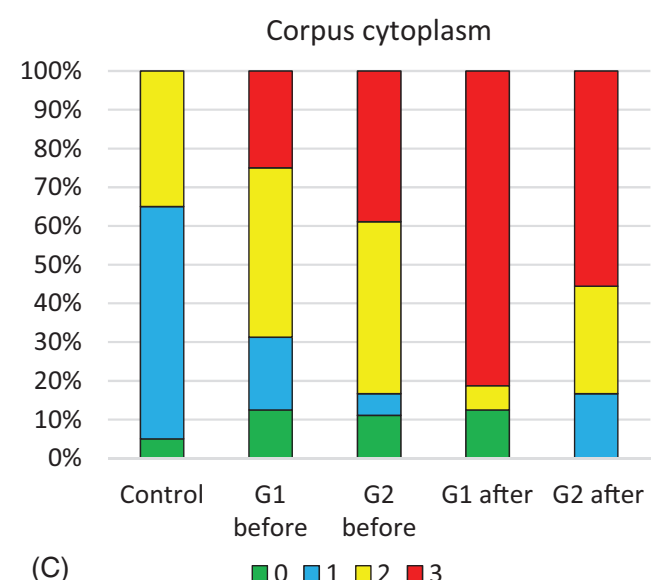

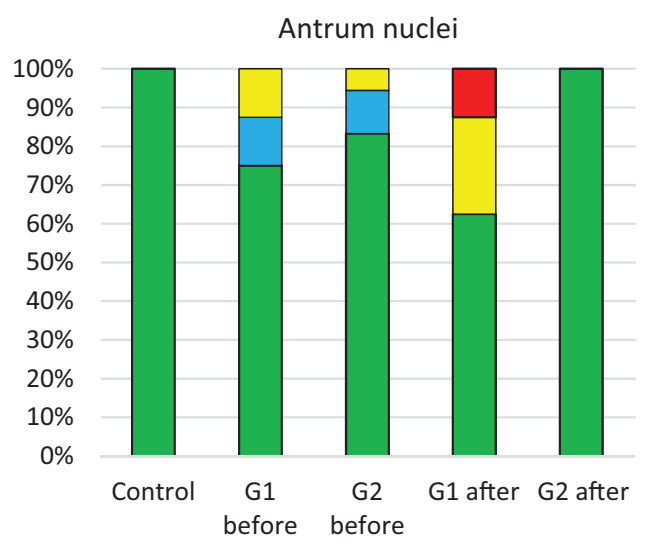

$\square 0 \square 1 \square 2 \square 3$

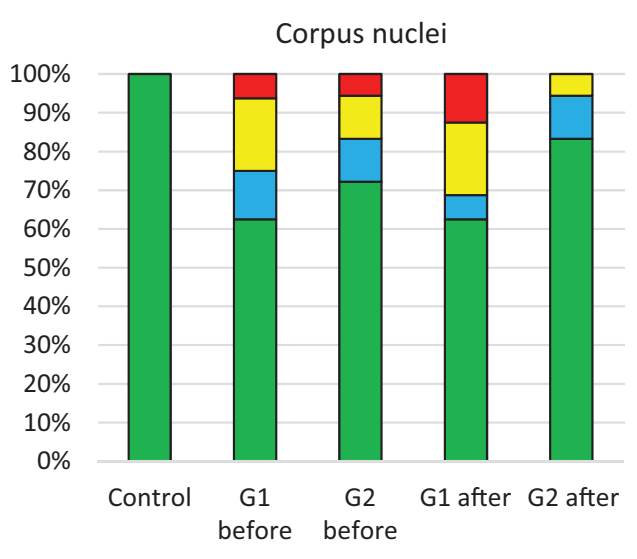

(D)

$\square 0 \square 1 \square 2 \square 3$

Figure 4. Semiquantitative evaluation of the accumulation of 4-hydroxynonenal-histidine adducts in cytoplasm and nuclei of glandular cells of gastric mucosa of antrum and corpus. Nonparametric Mann-Whitney $U$ test was used to compare the pattern of HNE-immunopositivity in studied patients (nonsignificant differences not indicated): (A) Control vs. G2 before $p<.05$, others $p>.05$; (B) Control vs. G1 before $p=.05$, Control vs. G1 after $p<.01, \mathrm{G} 2$ before vs. G1 after $p<.05, \mathrm{G} 1$ after vs. G2 after $p<.01$; (C) Control vs. G1 before $p<.01$, Control vs. G2 before $p<.001$, Control vs. G1 after $p<.001$, Control vs. G2 after $p<.001$, G1 before vs. G1 after $p<.001, \mathrm{G} 1$ before vs. $\mathrm{G} 2$ after $p<.05, \mathrm{G} 2$ before vs. $\mathrm{G} 1$ after $p<.01$; (D) Control vs. G1 before $p<.01$, Control vs. G2 before $p<.05$, Control vs. G1 after $p<.01$.

The HNE immunopositivity patterns of HNE-histidine immunohistochemical staining are presented as follows: before treatment (Figure $5(A, C)$ ), after standard treatment (Figure $5(B, D)$ ) and after standard treatment with AO supplementation (Figure 6).

\section{HRV parameters in DPU patients before and after treatment}

Time and frequency domain parameters of HRV were determined in all studied subjects and the results are summarised in Tables 6 and 7. DPU patients have increased heart rate (HR) and decreased HRV parameters (Table 6). After standard treatment further decrease in SDNN, RMSSD, and pNN50 $(p<.05)$ was observed, while HR did not change ( $p>.05$, Table 6$)$. In contrast, mild decrease in HR was statistically significant for the group 2 ( $p<.05$, Table 6$)$.

The results of determination of spectral (frequency domain) parameters showed similar changes to time domain indexes (Table 7). DPU patients of both groups before treatment had significantly lower TP, VLF, LF, and $\mathrm{HF}$, with higher LF/HF ratio in comparison to control group (Mann-Whitney $U$-test, $p<.05$ ). Likewise, after standard treatment further reduction of TP, VLF, $\mathrm{LF}$, and HF was observed $(p<.05$, Table 7). Patients of group 2, who were supplemented with AO, showed increase in most of spectral parameters of HRV, however, only for HF (indicating parasympathetic activity) statistical significance was obtained $(p<.05$, Table 7). Notably, all spectral parameters of HRV in group 2 after treatment were significantly higher than respective parameters of group $1(p<.05)$, and in cases of VLF and 
(A)

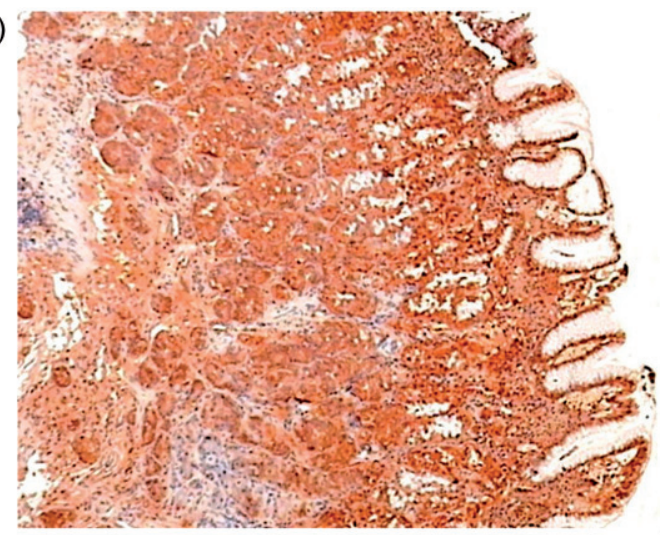

(x200)

(C)

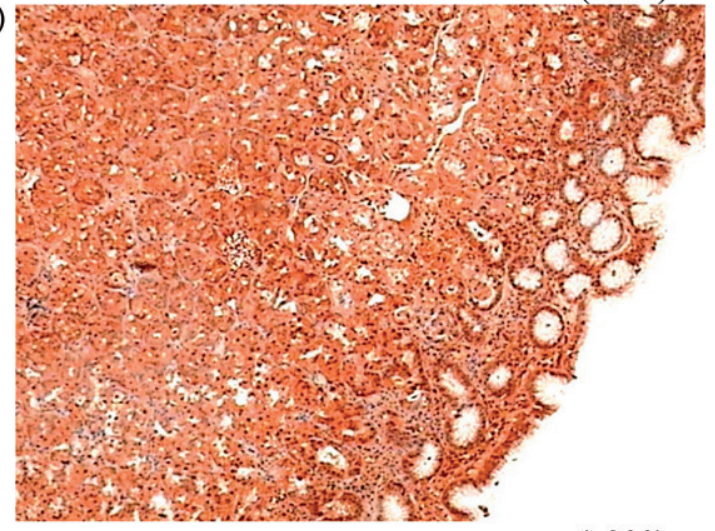

(B)

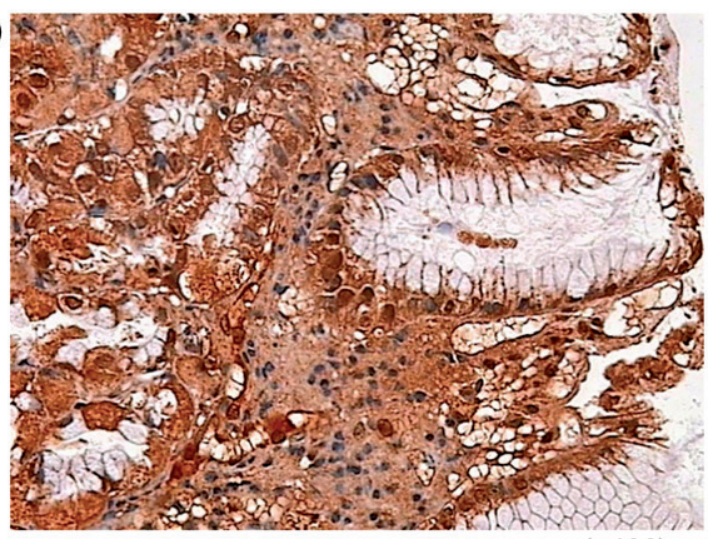

$(\mathrm{x} 400)$

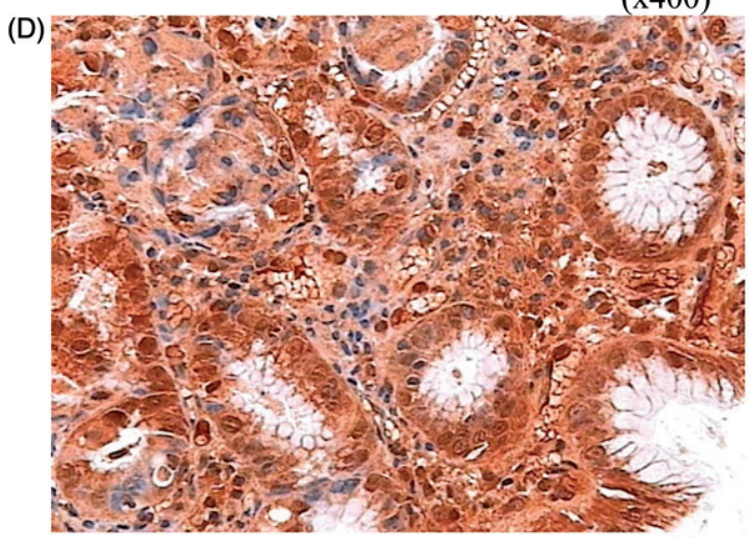

$(\mathrm{x} 400)$

Figure 5. Immunohistochemical determination of HNE-histidine conjugates before and after standard treatment. Images A and B reveal a marked accumulation of HNE-histidine adducts in cytoplasm and nuclei of majority of gastric glandular cells. Superficial and foveolar epithelium as well as lamina propria show significant HNE immunopositivity, however, the most significant accumulation is observed in glandular cells. C and D - the pattern of accumulation and distribution of HNE-histidine conjugates 4 weeks after end of eradication treatment is comparable with the pattern before treatment and in glandular epithelium grade of HNE immunopositivity is even greater.

HF reached or slightly exceeded the values of healthy subjects (Table 7). The effects of AO on HRV (improvement of parameters reflecting parasympathetic tone) were this consistent with the effects on HNE-histidine adducts accumulation in GM (reduction of HNE immunopositivity in group with $\mathrm{AO}$ supplementation in addition to standard treatment).

\section{Discussion}

The results of this study confirm the capabilities of supplementation of $\mathrm{AO}$ to modulate redox balance and reduce manifestations of OS in GM of patients with $H$. pylori-associated DPU in clinical conditions. It was demonstrated that elevated levels of HNE-histidine adducts in GM observed in acute stage of DPU in most of the patients remain after treatment at the high levels despite the evidence of clinical improvement (decrease or complete disappearance of clinical symptoms, effective ulcer healing, normalisation of GM histology, and eradication of $\mathrm{H}$. pylori), but $\mathrm{AO}$ supplementation additional to standard treatment, hence not influencing significantly clinical outcomes of DPU, causes reduction of OS manifestation within the study time frame. In addition to local effects in GM, a systemic effect in the form of HRV improvement was observed.

Helicobacter pylori is well-known pathogen colonising GM of billions of people worldwide and often causing chronic gastritis, peptic ulcers, gastric cancer, and a number of other health problems [36]. Ability to induce OS in stomach is well documented pathogenic property of this bacterium, and a growing body of evidence suggest that this effect may be systemic causing numerous extragastric manifestations of $H$. pylori [9,37-39]. Altered redox signalling plays a central role as a cause and consequence of acute and chronic inflammation, gastric carcinogenesis, dysregulation of prostaglandin metabolism, release of proinflammatory cytokines in bloodstream and causing low-grade inflammation, potentially contributing to the development of insulin resistance, endothelial dysfunction, and related problems [40-42]. 
(A)

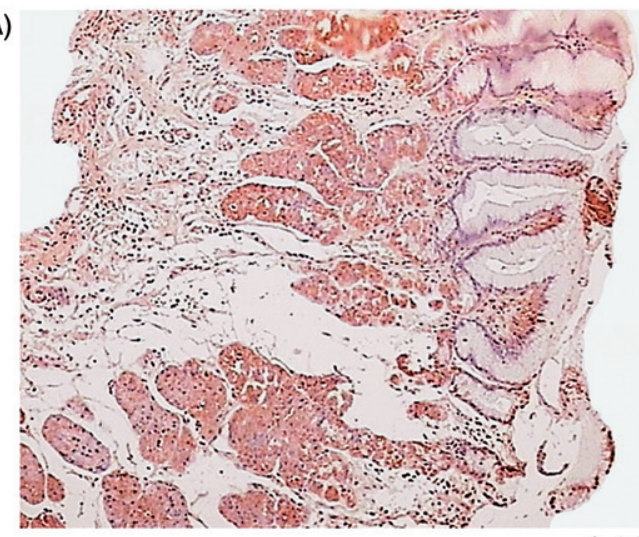

(C)

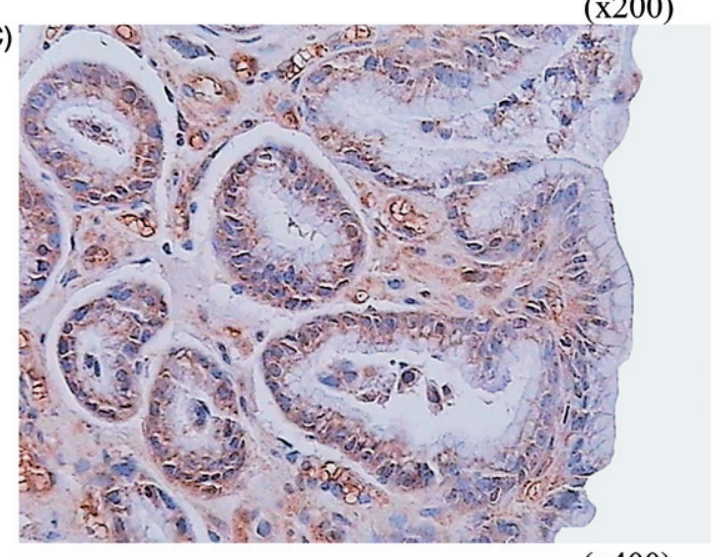

(B)

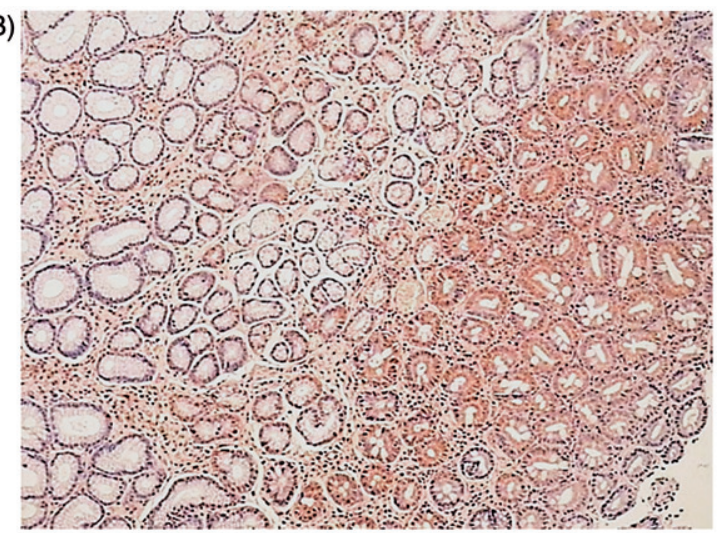

$(\mathrm{x} 200)$

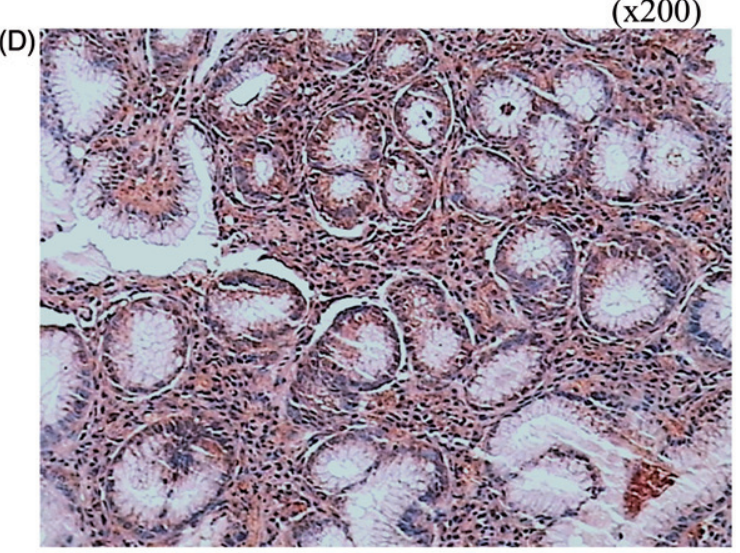

(x400)

Figure 6. Immunohistochemical determination of HNE-histidine conjugates after standard treatment with additional Amaranth oil supplementation. A, B moderate accumulation of HNE-histidine adducts predominantly in cytoplasm of glandular cells after 4 weeks of Amaranth oil supplementation, that started just after the end of eradication treatment. Nuclear accumulation of HNE-histidine adducts is not observed. The staining of superficial and foveolar epithelium and lamina propria shows absence of or low HNE immunopositivity. C, D - higher magnification confirms the findings from images A and B. There is the evidence of the reduction of HNE-histidine adducts accumulation in gastric mucosa compared to the condition before treatment and after standard treatment.

Table 6. Heart rate and time domain parameters of heart rate variability in DPU patients before and after treatment and apparently healthy subjects, median (quartile range).

\begin{tabular}{|c|c|c|c|c|}
\hline & Heart rate, beats/min. & SDNN, ms & RMSSD, ms & pNN50, \% \\
\hline Group 1 before treatment $(n=39)$ & $76.5(16.0)^{i}$ & $36.0(23.0)^{i}$ & $22.0(19.0)^{i}$ & $2.9(10.3)^{i}$ \\
\hline Group 1 after treatment $(n=39)$ & $73.1(11.1)^{\mathrm{h}}$ & $26.2(16.1)^{\mathrm{c}, \mathrm{i}}$ & $18.5(6.2)^{b, i}$ & $1.3(2.9)^{\mathrm{a}, \mathrm{i}}$ \\
\hline Group 2 before treatment $(n=36)$ & $75.9(11.0)^{\mathrm{h}}$ & $41.1(20.8)^{\mathrm{h}}$ & $29.0(20.1)^{\mathrm{h}}$ & $6.5(14.8)^{\mathrm{h}}$ \\
\hline Group 2 after treatment $(n=36)$ & $70.0(8.3)^{\mathrm{a}}$ & $43.2(5.8)^{h, e}$ & $33.0(4.4)^{g, e}$ & $14.0(10.9)^{g, e}$ \\
\hline Control group $(n=40)$ & $70.0(13.0)$ & $50.0(32.0)$ & $38.0(35.0)$ & $18.5(35.0)$ \\
\hline
\end{tabular}

${ }^{\mathrm{a}}$ The difference between before and after treatment within the groups significant, $p<.05$.

${ }^{\mathrm{b}}$ The difference between before and after treatment within the groups significant, $p<0.01$.

'The difference between before and after treatment within the groups significant, $p<.001$.

${ }^{\mathrm{e}}$ The difference between the parameters of groups after treatment significant, $p<.01$.

${ }^{9}$ The difference compared to control group significant, $p<.05$.

${ }^{\mathrm{h}}$ The difference compared to control group significant, $p<.01$.

'The difference compared to control group significant, $p<.001$.

Eradication of $H$. pylori, however, often does not cause instant effect and substantial time is needed to eliminate the consequences of $H$. pylori contamination as a risk factor for various disorders. For example, in case of gastric cancer successful $H$. pylori eradication significantly reduces risk of this common and often deadly neoplasm only after 8-10 years or more [5]. Molecular milieu of such an unusual "posteradication" persistence of OS is not completely understood and requires further in-depth analysis.

Immunohistochemistry is well recognised, advanced method of HNE determination in tissues and 
Table 7. Frequency domain parameters of heart rate variability in DPU patients before and after treatment and apparently healthy subjects, median (quartile range).

\begin{tabular}{|c|c|c|c|c|c|}
\hline & $\mathrm{TP}, \mathrm{ms}^{2} 10^{3}$ & VLF, $\mathrm{ms}^{2} \times 10^{3}$ & $\mathrm{LF}, \mathrm{ms}^{2} \times 10^{3}$ & $\mathrm{HF}, \mathrm{ms}^{2} \times 10^{3}$ & $\mathrm{LF} / \mathrm{HF}$ \\
\hline Group 1 before treatment $(n=39)$ & $1.64(1.99)^{i}$ & $0.70(0.92)$ & $0.48(0.47)^{i}$ & $0.25(0.45)^{i}$ & $1.38(1.56)^{\mathrm{h}}$ \\
\hline Group 1 after treatment $(n=39)$ & $0.89(1.04)^{c, i}$ & $0.47(0.26)^{c, i}$ & $0.28(0.33)^{c, i}$ & $0.15(0.11)^{c, i}$ & $1.51(1.92)^{i}$ \\
\hline Group 2 before treatment $(n=36)$ & $2.16(2.09)^{\mathrm{g}}$ & $0.96(0.74)$ & $0.64(0.75)^{\mathrm{g}}$ & $0.44(0,73)^{g}$ & $2.12(1.52)$ \\
\hline Group 2 after treatment $(n=36)$ & $2.62(0.35)^{\mathrm{f}, \mathrm{g}}$ & $0.84(0.11)^{f}$ & $0.71(0.11)^{\mathrm{f}, \mathrm{g}}$ & $0.97(0.13)^{\mathrm{a}, \mathrm{f}, \mathrm{h}}$ & $1.90(0.26)^{\mathrm{a}, e, g}$ \\
\hline Control group $(n=40)$ & $3.04(2.75)$ & $0.85(1.29)$ & $0.85(0.76)$ & $0.86(1.15)$ & $0.95(1.12)$ \\
\hline
\end{tabular}

${ }^{a}$ The difference between before and after treatment within the groups significant, $p<.05$.

'The difference between before and after treatment within the groups significant, $p<.001$.

${ }^{\mathrm{e}}$ The difference between the parameters of groups after treatment significant, $p<.01$.

${ }^{\mathrm{f}}$ The difference between the parameters of groups after treatment significant, $p<.001$.

The difference compared to control group significant, $p<.05$.

${ }^{\mathrm{h}}$ The difference compared to control group significant, $p<.01$.

'The difference compared to control group significant, $p<.001$.

significantly contributed to the establishment of the role of HNE in health and disease. It became evident by immunohistochemical analysis that HNE-protein adducts moderately accumulate not only in pathological but also in physiological conditions being more pronounced under onset of chronic or acute OS, both due to exogenous stressors (toxins, microbial agents, hypo/hyperoxia) and to the endogenous pathophysiological processes such as inflammation related to carcinogenesis and/or defence against cancer etc. $[15,16]$ Hence, the HNE-immunohistochemistry allows us to evaluate accumulation and distribution of HNE-protein adducts, that contributes to improvement of the quality and precision of the analysis [12].

Amaranth oil was previously demonstrated to have redox-modulating properties and efficiency in relieving of OS manifestation in high-level competitive athletes [21], diabetic, coronary artery disease, and hypertension patients [32]. Animal studies also showed substantial therapeutic potential of $A O$ in treatment of hypercholesterolemia [43], antitumour activity [44] etc. Considering unique chemical composition and high content of squalene, polyunsaturated fatty acids, tocopherols, and tocotrienols, potential redox-modulating activity of $\mathrm{AO}$ could be attributed to its metabolically active components that, in case of gastroduodenal diseases, could be directly delivered to the site when administered orally [32].

The effects of $A O$ in our clinical setup were consistent with the previous findings from the literature described above. In fact, standard treatment of DPU directed on $H$. pylori eradication causes dramatic improvement of clinical symptoms, healing of the ulcers, and reducing inflammation (Tables 1, 2, and 5), however, the evidence from HNE-immunohistochemistry indicates persistence of OS in GM, despite successful H. pylori eradication (Figures 2-6). Obviously, eradication of $H$. pylori is a major contributor to reduction of number of inflammatory cells in mucosa samples (Table 5), and, to some extent, AO may also contribute to this effect (significant reduction of monocytes in AO-treated group compared to standard treatment, Table 5). However, this is probably not the major effect of $\mathrm{AO}$, since the inflammation was dramatically reduced in both groups after treatment, while reduction of HNE-adducts accumulation was observed mainly in group 2. Despite individual variability of the patients' response to treatment, the $A O$ provided significant reduction of the HNE-histidine adducts in GM and therefore local reduction of OS manifestations. Thus, anti-OS effect of AO, at least in part, seems to be independent on anti-inflammatory effect.

However, the effects of $A O$ are not limited to local impact on GM. It was shown that $A O$ supplementation, in contrast to standard treatment, improves HRV in DPU patients after $H$. pylori eradication. On the other hand, cholinergic effects of $A O$ at systemic level, in turn, may also contribute to anti-inflammatory effects at the level of gastric mucosa $[24,25]$ due to improvement of endothelial function and local blood flow that is known to have major effect on ulcer healing [45].

Since $A O$ is a relatively complex mixture of different lipid components, underlying mechanisms of its redoxmodulatory effects are not clearly known. There are not many studies addressing this issue in the literature, but there are reasons to consider $A O$ as a mild prooxidant factor and its effects as hormetic response of respective tissues/organism [46]. Considering sensitivity of HRV to metabolic conditions in the organism [19], positive effects of AO supplementation on HRV are not surprising.

There are several important limitations in this study. First, immunohistochemistry is considered to be highly specific for antigen epitope (in this case HNE-histidine adducts), but many factors such as quality of reagents, adequate storage of samples, etc., could reduce reliability of the specimens processed [12]. In order to address these issues we paid particular attention to acquire, handle and store samples according to the optimised protocol. Histological and immunohistochemical evaluations were performed by experienced pathologists, 
without previous knowledge of the character of the specimens, while the same standard reagents were used for the simultaneous processing of the specimens. Although we did not use confocal microscopy in current study, the term "nuclear" accumulation of HNEhistidine adducts refers to nuclear or perinuclear HNE-immunopositivity, which reflects higher degree of oxidative stress, since nuclei are very well protected against oxidative damage [47], while such accumulation of the HNE-histidine adducts was revealed by confocal microscopy in previous animal study to occur predominantly near nuclear membrane [48]. Finally, $\mathrm{AO}$ is a complex mixture of different lipid compounds, therefore, it is impossible to determine directly which of them had major beneficial effects in our study. Hence, we also could not measure the levels of potentially active substances in blood, which might have been involved in systemic effects of AO reflected by the HRV observations.

AO supplementation is not the only intervention capable to reduce local and/or systemic manifestations of OS and the mechanism could be also quite different. For example, positive effects of interval hypoxic training [49], intermittent fasting/caloric restriction [29], exercise [50], antiulcer activity of highly purified calf haemodialisate [45], numerous redox-active fruits [51], and vegetables [52] that may activate oxygen metabolism may physiologically stimulate redox signalling and cause local and systemic metabolic effects.

\section{Conclusions}

The study has shown that supplementation of Amaranth oil in addition to standard anti-H. pylori treatment significantly reduced accumulation of HNE in GM and increased HRV in DPU patients. Improvement of clinical, endoscopic and histological parameters, and successful ulcer healing was observed in both groups and no statistically significant difference was detected. Persistence of accumulation of the HNE-histidine adducts after standard anti-DPU treatment requires additional therapeutic approaches aiming to follow modern principles of integrative medicine and reduce manifestations of chronic oxidative stress, as was observed in our patients after beneficial use of AO.

\section{Acknowledgements}

The study was supported by COST Actions B35 "LPO-lipid peroxidation associated disorders", CM1001 "Chemistry of non-enzymatic protein modification - modulation of protein structure and function", "BM1203 "EU-ROS" "New concepts and views in redox biology and oxidative stress research", CA16112 "Personalized Nutrition in aging society: redox control of major age-related diseases", A.C. was supported by the Georg Forster (HERMES) Scholarship from Alexander Von Humboldt Foundation (Bonn, Germany).

\section{Disclosure statement}

The authors report no conflicts of interest. The authors alone are responsible for the content and writing of this article.

\section{Funding}

Alexander von Humboldt-Stiftung; European Cooperation in Science and Technology.

\section{ORCID}

Andriy Cherkas (iD) http://orcid.org/0000-0002-6652-6983

Morana Jaganjac (iD http://orcid.org/0000-0001-5051-1843

Lidija Milkovic (D) http://orcid.org/0000-0002-4484-039X

Orest Abrahamovych (iD http://orcid.org/0000-0001-68626809

Ostap Yatskevych (D) http://orcid.org/0000-0001-5146-2553

\section{References}

1. Najm WI. Peptic ulcer disease. Prim Care 2011;38(3): 383-94, vii.

2. Franceschi F, Gasbarrini A, Polyzos SA, Kountouras J. Extragastric diseases and Helicobacter pylori. Helicobacter 2015;20(Suppl1):40-46.

3. Kountouras J, Boziki $M$, Polyzos SA, Katsinelos $P$, Gavalas $E$, Zeglinas $C$, et al. Impact of reactive oxygen species generation on Helicobacter pylori-related extragastric diseases: a hypothesis. Free Radic Res 2017; 51:73-79.

4. Suki M, Leibovici Weissman $Y$, Boltin D, Itskoviz D, Tsadok Perets T, Comaneshter D, et al. Helicobacter pylori infection is positively associated with an increased $\mathrm{BMI}$, irrespective of socioeconomic status and other confounders: a cohort study. Eur J Gastroenterol Hepatol 2018;30:143-148.

5. O'Connor A, O'Morain CA, Ford AC. Population screening and treatment of Helicobacter pylori infection. Nat Rev Gastroenterol Hepatol 2017;14:230-240.

6. Dogan Z, Sarikaya M, Ergul B, Filik L. The effect of Helicobacter pylori eradication on insulin resistance and $\mathrm{HbA} 1 \mathrm{c}$ level in people with normal glucose levels: a prospective study. Biomed Pap Med Fac Univ Palacky Olomouc Czech Repub 2015;159:242-245.

7. Handa O, Naito Y, Yoshikawa T. Helicobacter pylori: a ROS-inducing bacterial species in the stomach. Inflamm Res 2010;59:997-1003.

8. Cherkas A, Eckl P, Gueraud F, Abrahamovych O, Serhiyenko V, Yatskevych $O$, et al. Helicobacter pylori in sedentary men is linked to higher heart rate, sympathetic activity, and insulin resistance but not inflammation or oxidative stress. Croat Med J 2016;57: 141-149. 
9. Waluga $M$, Kukla $M$, Żorniak $M$, Bacik A., Kotulski R. From the stomach to other organs: Helicobacter pylori and the liver. World J Hepatol 2015;7:2136-2146.

10. Zarkovic N, Cipak A, Jaganjac M, Borovic S, Zarkovic K. Pathophysiological relevance of aldehydic protein modifications. J Proteom 2013;92:239-247.

11. Zarkovic N. 4-hydroxynonenal as a bioactive marker of pathophysiological processes. Mol Aspects Med 2003; 24(4-5):281-291.

12. Zarkovic K, Jakovcevic A, Zarkovic N. Contribution of the HNE-immunohistochemistry to modern pathological concepts of major human diseases. Free Radic Biol Med 2017;111:110-126.

13. Weber D, Milkovic L, Bennett SJ, Griffiths HR, Zarkovic $\mathrm{N}$, Grune T. Measurement of HNE-protein adducts in human plasma and serum by ELISA-Comparison of two primary antibodies. Redox Biol 2013;1:226-233.

14. Cipak Gasparovic A, Zarkovic N, Zarkovic K, Semen K, Kaminskyy D, Yelisyeyeva O, Bottari SP. Biomarkers of oxidative and nitro-oxidative stress: conventional and novel approaches. Br J Pharmacol 2017;174:1771-1783.

15. Yelisyeyeva O, Cherkas A, Zarkovic K, Semen K, Kaminskyy D, Waeg G, Zarkovic N. The distribution of 4-hydroxynonenal-modified proteins in gastric mucosa of duodenal peptic ulcer patients. Free Radic Res 2008;42:205-211.

16. Cherkas A, Yelisyeyeva O, Semen K, Zarkovic K, Kaminskyy D, Gasparovic AC, et al. Persistent accumulation of 4-hydroxynonenal-protein adducts in gastric mucosa after Helicobacter pylori eradication. Coll Antropol 2009;33:815-821.

17. Sgouras DN, Trang TT, Yamaoka Y. Pathogenesis of Helicobacter pylori infection. Helicobacter 2015; 20(Suppl1):8-16.

18. Cherkas A, Golota S, Guéraud F, Abrahamovych $\mathrm{O}$, Pichler C, Nersesyan A, et al. A Helicobacter pylori-associated insulin resistance in asymptomatic sedentary young men does not correlate with inflammatory markers and urine levels of 8-iso-PGF2-alpha or 1,4dihydroxynonane mercapturic acid. Arch Physiol Biochem 2017:1-11.

19. Cherkas A, Yatskevych O. The amplitude of heart rate oscillations is dependent on metabolic status of sinoatrial node cells. OA Med Hypothesis 2014;2:1-8.

20. Cherkas A, Abrahamovych O, Golota S, Nersesyan A, Pichler C, Serhiyenko V, et al. The correlations of glycated hemoglobin and carbohydrate metabolism parameters with heart rate variability in apparently healthy sedentary young male subjects. Redox Biol 2015;5:301-307.

21. Yelisyeyeva O, Cherkas A, Semen K, Kaminskyy D, Lutsyk A. Study of aerobic metabolism parameters and heart rate variability and their correlations in elite athletes: a modulatory effect of amaranth oil. Clin Exp Med J 2009;3:293-307.

22. Deding U, Ejlskov L, Grabas MP, Nielsen BJ, TorpPedersen C, Boggild H. Perceived stress as a risk factor for peptic ulcers: a register-based cohort study. BMC Gastroenterol 2016;16:140.

23. Abrahamovych $O$, Cherkas A, Abrahamovych $U$, Abrahamovych $M$, Serhiyenko V. Heart rate variability: physiological bases, clinical importance and peculiarities in patients with peptic ulcer and after resection of stomach. Lviv: Danylo Halytskyi Lviv National Medical University; 2014.

24. Zila I, Mokra D, Kopincova J, Kolomaznik M, Javorka M, Calkovska A. Vagal-immune interactions involved in cholinergic anti-inflammatory pathway. Physiol Res 2017;66(Suppl2):S139-SS145.

25. Hoover DB. Cholinergic modulation of the immune system presents new approaches for treating inflammation. Pharmacol Ther 2017;179:1-16.

26. Steinhorn B, Sartoretto JL, Sorrentino A, Romero N, Kalwa $\mathrm{H}$, Abel ED, Michel T. Insulin-dependent metabolic and inotropic responses in the heart are modulated by hydrogen peroxide from NADPH-oxidase isoforms NOX2 and NOX4. Free Radic Biol Med 2017;113:16-25.

27. Contreras C, Nogueiras R, Diéguez C, Rahmouni K, Lopez M. Traveling from the hypothalamus to the adipose tissue: the thermogenic pathway. Redox Biol 2017;12:854-863.

28. Gao L, Zimmerman MC, Biswal S, Zucker IH. Selective Nrf2 gene deletion in the rostral ventrolateral medulla evokes hypertension and sympathoexcitation in mice. Hypertension 2017;69:1198-1206.

29. Cherkas A, Golota S. An intermittent exhaustion of the pool of glycogen in the human organism as a simple universal health promoting mechanism. Med Hypotheses 2014;82:387-389.

30. Klotz LO, Sánchez-Ramos C, Prieto-Arroyo I, Urbanek P, Steinbrenner $\mathrm{H}$, Monsalve $\mathrm{M}$. Redox regulation of FoxO transcription factors. Redox Biol 2015;6:51-72.

31. Tang $Y$, Tsao R. Phytochemicals in quinoa and amaranth grains and their antioxidant, anti-inflammatory, and potential health beneficial effects: a review. Mol Nutr Food Res 2017;61.

32. Martirosyan DM, Miroshnichenko LA, Kulakova SN, Pogojeva AV, Zoloedov VI. Amaranth oil application for coronary heart disease and hypertension. Lipids Health Dis 2007;6:1.

33. Hołubiuk $\measuredangle$, Imiela J. Diet and Helicobacter pylori infection. Prz Gastroenterol 2016;11:150-154.

34. Waeg G, Dimsity G, Esterbauer H. Monoclonal antibodies for detection of 4-hydroxynonenal modified proteins. Free Radic Res 1996;25:149-159.

35. Cherkas A, Zhuraev R. A marked decrease in heart rate variability in Marfan syndrome patients with confirmed FBN1 mutations. Cardiol J 2016;23:23-33.

36. Mentis A, Lehours P, Mégraud F. Epidemiology and Diagnosis of Helicobacter pylori infection. Helicobacter 2015;20(Suppl1):1-7.

37. Chen LW, Chien CY, Yang KJ, Kuo SF, Chen CH, Chien RN. Helicobacter pylori infection increases insulin resistance and metabolic syndrome in residents younger than 50 years old: A community-based study. PLoS One 2015;10:e0128671.

38. Buzás GM. Metabolic consequences of Helicobacter pylori infection and eradication. World J Gastroenterol 2014;20:5226-5234.

39. Lin Y, Obata Y, Kikuchi S, Tamakoshi A, Iso H, JACC Study Group. Helicobacter pylori infection and risk of death from cardiovascular disease among the Japanese population: a nested case-control study within the JACC Study. J Atheroscler Thromb 2015;22:1207-1213. 
40. Yeniova AO, Uzman M, Kefeli A, Basyigit S, Ata N, Dal K, Guresci S, Nazligul Y. Serum 8 hydroxydeoxyguanosine and cytotoxin associated gene $A$ as markers for Helicobacter pylori infection. Asian Pac J Cancer Prev 2015;16:5199-5203.

41. Okin D, Medzhitov R. The effect of sustained inflammation on hepatic mevalonate pathway results in hyperglycemia. Cell 2016;165:343-356.

42. Pohjanen VM, Koivurova OP, Niemelä SE, Karttunen RA, Karttunen TJ. Role of Helicobacter pylori and interleukin 6-174 gene polymorphism in dyslipidemia: a case-control study. BMJ Open 2016;6:e009987.

43. Berger A, Gremaud G, Baumgartner $M$, Rein D, Monnard I, Kratky E, et al. Cholesterol-lowering properties of amaranth grain and oil in hamsters. Int J Vitam Nutr Res 2003;73:39-47.

44. lelisieieva OP, Kamins'ky $\breve{~ D V}$, Cherkas AP, Ambarova LI, Vyshemyrs'ka LD, Dzhura OR, et al. Characteristics of amaranth oil effect on the antioxidant system of the liver and blood in mice with malignant lymphoma growth. Ukr Biokhim Zh 2006;78:117-123.

45. Stelmakh A, Abrahamovych O, Cherkas A. Highly purified calf hemodialysate (actovegin(R)) may improve endothelial function by activation of proteasomes: a hypothesis explaining the possible mechanisms of action. Med Hypotheses 2016;95:77-81.

46. Guéraud F, Taché S, Steghens JP, Milkovic L, BorovicSunjic S, Zarkovic N, et al. Dietary polyunsaturated fatty acids and heme iron induce oxidative stress biomarkers and a cancer promoting environment in the colon of rats. Free Radic Biol Med 2015;83:192-200.

47. Jung $T$, Engels $M$, Kaiser B, Poppek D, Grune $T$. Intracellular distribution of oxidized proteins and proteasome in HT22 cells during oxidative stress. Free Radic Biol Med 2006;40:1303-1312.

48. Marquez-Quiñones A, Cipak A, Zarkovic K, FattelFazenda S., Villa-Trevino S, Waeg G, et al. HNE-protein adducts formation in different pre-carcinogenic stages of hepatitis in LEC rats. Free Radic Res 2010;44: 119-127.

49. Semen KO, Yelisyeyeva OP, Kaminskyy DV, Cherkas AP, Zarkovic K, Lutsyk $O$, et al. Interval hypoxic training in complex treatment of Helicobacter pyloriassociated peptic ulcer disease. Acta Biochim Pol 2010;57:199-208.

50. Radak Z, Ishihara K, Tekus E, Boldogh I, Koltai E. Exercise, oxidants, and antioxidants change the shape of the bell-shaped hormesis curve. Redox Biol 2017; 12:285-290.

51. Park YS, Namiesnik J, Vearasilp K, Leontowicz $H$, Leontowicz $M$, Barasch $D$, et al. Bioactive compounds and the antioxidant capacity in new kiwi fruit cultivars. Food Chem 2014;165:354-361.

52. Kim HG, Bae JH, Jastrzebski Z, Cherkas A, Heo BG, Gorinstein S, Ku YG. Binding, antioxidant and anti-proliferative properties of bioactive compounds of sweet paprika (Capsicum annuum L.). Plant Foods Hum Nutr 2016;71:129-136. 